\title{
Observer based synchronization of chaotic systems
}

\author{
Ömer Morgül* and Ercan Solak \\ Department of Electrical and Electronics Engineering, Bilkent University, 06533, Bilkent, Ankara, Turkey
}

(Received 22 April 1996; revised manuscript received 9 August 1996)

\begin{abstract}
We show that the synchronization of chaotic systems can be achieved by using the observer design techniques which are widely used in the control of dynamical systems. We show that local synchronization is possible under relatively mild conditions and global synchronization is possible if the chaotic system can be transformed into a special form. We also give some examples including the Lorenz, the Rössler systems, and Chua's oscillator which are known to exhibit chaotic behavior, and show that in these systems synchronization by using observers is possible. [S1063-651X(96)10511-0]
\end{abstract}

PACS number(s): 05.45.+b

\section{INTRODUCTION}

The concept of synchronization of chaotic systems may seem somewhat paradoxical since in such systems solutions starting from arbitrary close initial conditions quickly diverge and become uncorrelated. However, recently it has been shown that such synchronization is possible, see e.g., [1-3], and this subject then received a great deal of attention among scientists in many fields [1-9]. One of the motivations for synchronization is the possibility of sending messages through chaotic systems for secure communication [4-6]. Such synchronized systems usually consist of two parts: a generator of chaotic signals (drive system), and a receiver (response system). The response system is usually a duplicate of a part (or the whole) of the drive system. A chaotic signal generated by the drive system may be used as an input in the response system to synchronize the common signals of both systems, see e.g., [2]. After the synchronization, one may add the message to the chaotic signal used for synchronization, and under certain conditions one may recover the message from the signals of the response system [4]. We note that once the chaotic "drive" system is given, most of the synchronization schemes proposed in the literature do not give a systematic procedure to determine the "response" system and the drive signal. Hence most of these schemes depend on the choice of the drive system and could not be easily generalized to an arbitrary chaotic drive system.

A related problem encountered in the systems and control theory is the estimation of the states of a dynamical system by using another system, called an "observer." The theory of the design of observers, although not fully exploited, is a relatively well-studied branch of system theory and is widely used in the state feedback control of dynamical systems [1016]. In this paper our aim is to show that this existing theory of observers may naturally be used in the relatively new field of synchronization of chaotic systems. In this approach, once the drive system is given, the response system could be chosen in the observer form, and the drive signal should be chosen accordingly so that the drive system satisfies certain conditions. Under some relatively mild conditions, local or

\footnotetext{
*Fax: 90-312-266 41 26;
}

Electronic address: morgul@bilkent.edu.tr global synchronization of drive and observer systems can be guaranteed. Hence this synchronization scheme offers a systematic procedure, independent of the choice of the drive system. It is our belief that the interaction between these fields may be beneficial for both of the fields.

This paper is organized as follows. In Sec. II we present some basic material for the design of observers and show that local synchronization may be possible under certain conditions, which are not very restrictive. We consider the Lorenz and Rössler systems and show that for these systems local synchronization may be possible by using the observers. We also show that some of the existing schemes for synchronization (e.g., [2,3]) are related to the observer based synchronization. We also show that the proposed synchronization scheme is robust with respect to measurement noise. In Sec. III we consider a special form called the Brunowsky canonical form and by using the result of [12] show that if the chaotic system can be transformed into this form, global synchronization is possible. We also show that some of the chaotic systems (e.g., the Rössler system and Chua's oscillator) can be transformed into this form. In Sec. IV we present some numerical simulation results and finally, in Sec. $\mathrm{V}$, we give some concluding remarks.

\section{FULL ORDER OBSERVER}

We begin with the definition of observability for a linear system, which plays an important role in modern control theory. Consider the following linear system:

$$
\dot{u}=A u, \quad y=C u,
$$

where $A \in \mathbb{R}^{n \times n}, C \in \mathbb{R}^{m \times n}$ are constant matrices, $y$ is called the "output" of the system. The problem of observability is related to the computation of initial condition $u(0) \in \mathbb{R}^{n}$ by only observing the output $y(\cdot)$ over an interval of time.

Definition: (Observability) Consider the system described by (1). Two states $u_{0}$ and $u_{1}$ are said to be distinguishable if $y\left(t, u_{0}\right) \neq y\left(t, u_{1}\right)$ for some $t \geqslant 0$, where $y\left(t, u_{i}\right)=C e^{A t} u_{i}$, is the output $y(t)$ corresponding to the initial condition $u(0)=u_{i}, i=1,2$. The system given by (1) [or, in short, the pair $(C, A)]$ is said to be observable if all distinct states are distinguishable (see, e.g., $[14,13,15])$.

We next state the following well-known fact. 
Theorem 1: Consider the system given by (1). Then the following are equivalent:

(i) The pair $(C, A)$ is observable.

(ii) The following rank condition is satisfied:

$$
\operatorname{rank}\left(\begin{array}{c}
C \\
C A \\
\cdot \\
\cdot \\
\cdot \\
C A^{n-1}
\end{array}\right)=n \text {. }
$$

(iii) The following rank condition is satisfied:

$$
\operatorname{rank}\left(\begin{array}{c}
\lambda I-A \\
C
\end{array}\right)=n, \quad \forall \lambda \in \mathbf{C} .
$$

(iv) For any polynomial $p(\lambda)=\lambda^{n}+a_{1} \lambda^{n-1}+\cdots+$ $a_{n-1} \lambda+a_{n}, a_{i} \in \mathbb{R}, i=1,2, \ldots, n$, there exists a constant matrix $K \in \mathbb{R}^{n \times m}$ such that $\operatorname{det}(\lambda I-A+K C)=p(\lambda)$.

Proof: $\quad$ See e.g., [13], p. 80, p. 136, and [15], p. 61.

Consider the nonlinear system given below

$$
\dot{u}=A u+g(u), \quad y=C u,
$$

where $A \in \mathbb{R}^{n \times n}$ and $C \in \mathbb{R}^{m \times n}$ are constant matrices, $g$ : $\mathrm{R}^{n} \rightarrow \mathrm{R}^{n}$ is a differentiable function. Assume that $g$ satisfies the following Lipschitz condition:

$$
\left\|g\left(u_{1}\right)-g\left(u_{2}\right)\right\| \leqslant L\left\|u_{1}-u_{2}\right\|, \quad \forall u_{1}, u_{2} \in \mathbb{R}^{n},
$$

where $L>0$ is a Lipschitz constant and $\|\cdot\|$ is the standard Euclidean norm in $\mathrm{R}^{n}$. We will use a technique proposed in [10] for the observer design. We assume that the pair $(C, A)$ is observable. Now choose the matrix $K \in \mathbb{R}^{n \times m}$ such that $A_{c}=A-K C$ is a stable matrix, which is always possible since the pair $(C, A)$ is observable, see Theorem 1 . Then for any symmetric and positive definite matrix $Q \in \mathbb{R}^{n \times n}$ there exists a symmetric and positive definite matrix $P \in \mathbb{R}^{n \times n}$ such that the following well-known Lyapunov matrix equation is satisfied:

$$
A_{c}^{T} P+P A_{c}=-Q
$$

where the superscript $T$ denotes the transpose [14]. For the system given by (4), we choose the following "observer", equation:

$$
\dot{\hat{u}}=A \hat{u}+g(\hat{u})+K C(u-\hat{u}),
$$

which is known as the full order observer or the Luenberger observer [12]. Note that the signals $y=C u$ and $\hat{u}$ are available, hence, the observer given by (7) is implementable. Let us define the error of observation as $e=u-\hat{u}$. By using (4) and (7) we obtain the following error equation:

$$
\dot{e}=(A-K C) e+g(u)-g(\hat{u}) .
$$

Now let the symmetric and positive definite matrices $P$ and $Q$ satisfy (6). By using the Lyapunov function $V=e^{T} P e$, it can be shown that if

$$
L<\frac{\lambda_{\min }(Q)}{2 \lambda_{\max }(P)},
$$

then we have the following:

$$
\|e(t)\| \leqslant M e^{-\alpha t}\|e(0)\|,
$$

where

$$
M=\left[\frac{\lambda_{\max }(P)}{\lambda_{\min }(P)}\right]^{1 / 2}, \quad \alpha=\frac{\lambda_{\min }(Q)}{2 \lambda_{\max }(P)}-L>0,
$$

and $\lambda_{\max }(T), \lambda_{\min }(T)$ denote the maximum and minimum eigenvalues of a symmetric matrix $T$, respectively. For details, see [10], and for a survey on observer theory, see [11].

In the application of the observer theory given above, the main difficulty is in the Lipschitz property given by (5), which should be satisfied globally. But if (5) is satisfied, then the observer given by (7) works globally, i.e., for all $e(0) \in \mathbb{R}^{n}$, provided that (9) is satisfied. We may relax this condition as follows, but then the result (10) may hold locally, i.e., in a compact region for $e(0)$.

Lemma 1: Consider the systems given by (4) and (7). Assume that the pair $(C, A)$ is observable, $g: \mathbb{R}^{n} \rightarrow \mathbb{R}^{n}$ is differentiable and that the following is satisfied:

$$
\lim _{u \rightarrow 0}\|D g(u)\|=0
$$

where $D g(\cdot)$ denotes the Jacobian of $g$. Then there exist a matrix $K \in \mathbb{R}^{n \times m}$ and a real number $r>0$ such that (10) holds if $\|e(0)\| \leqslant r$ and $\|u(t)\| \leqslant r, \forall t \geqslant 0$.

Proof: Choose a matrix $K \in \mathbb{R}^{n \times m}$ such that $A_{c}=A-K C$ is a stable matrix, and choose the symmetric and positive definite matrices $P$ and $Q$ which satisfy (6). For $R>0$, we may take $L>0$ in (5) as

$$
L=\sup \{\|D g(u)\| \mid\|u\| \leqslant R\} .
$$

Now choose $R>0$ such that $L>0$ given by (12) satisfies (9). Note that since (11) holds, this is always possible. Let $\|\hat{u}(0)\| \leqslant r_{1}$ and $\|u(t)\| \leqslant r_{2}, \forall t \geqslant 0$ for some $r_{1}>0$ and $r_{2}>0$. By using the Bellman-Gronwall inequality, (5) and (7) (see e.g., $[14,16]$, it can be proven that if $r_{1}$ and $r_{2}$ are sufficiently small, then $\hat{u}(t)$ remains bounded as $\|\hat{u}(t)\| \leqslant r_{3}$ for some $r_{3}>0$. Moreover, as $r_{1} \rightarrow 0$ and $r_{2} \rightarrow 0$, we have $r_{3} \rightarrow 0$ as well. Hence there exists a $r>0$ satisfying $R>r$ such that if $\|u(t)\| \leqslant r$ and $\|e(0)\| \leqslant r$, then we have $\|\hat{u}(t)\| \leqslant R$, hence the Lipschitz constant $L$ given by (12) remains valid $\forall t \geqslant 0$. Then it follows that (10) remains valid $\forall t \geqslant 0$.

Remark 1: Lemma 1 states that if (11) holds, if the initial error $e(0)$ is sufficiently small and if $u(t)$ remains in a sufficiently small region, then for the observer given by (7), the estimate given by (10) is satisfied. Since in chaotic systems the solutions which are of interest to us are bounded, this lemma might be used for local synchronization. However, the lemma does not provide an estimate on the bound $r$. Note that the condition given by (11) is less stringent than the Lipschitz condition (5) and (9). In applications, the differential equation given by (4) is obtained by linearization of a nonlinear system around an equilibrium point. In such cases, the function $g$ necessarily contains at least second order terms, hence (11) is automatically satisfied. 
The observer design technique given above assumes that an output $y(\cdot)$ which is transmitted to the observer is available, see (4) and (7). However, in chaotic systems such an output is not given a priori and has to be chosen as a part of the observer design procedure. In view of the observer theory given above, obviously one should choose the output as in (4) so that the pair $(C, A)$ is observable. (The observability condition may be changed to a "detectability" condition, which is weaker than observability. See Remark 3 and the Example 1 below). Moreover, for practical considerations, the dimension $m$ should be as low as possible, since $y(t) \in \mathbb{R}^{m}$ is the signal transmitted to the observer. Case $m=1$ is possible under certain conditions, which are given below. Note that a matrix $A \in \mathbb{R}^{n \times n}$ is called cyclic if in its Jordan canonical form, for each eigenvalue of $A$ there exists one and only one Jordan block. This guarantees that $\operatorname{rank}\left(\lambda_{i} I-A\right)=n-1$ for any eigenvalue $\lambda_{i}$ of $A$.

Lemma 2: Let $A \in \mathbb{R}^{n \times n}$ be given. Then there exists a vector $C^{T} \in \mathrm{R}^{n}$ such that $(C, A)$ is observable if and only if $A$ is cyclic.

Proof: This could easily be proven by using (3). Moreover, let $\lambda_{1}, \ldots, \lambda_{p}$ be the eigenvalues and $v_{1}, \ldots, v_{p}$ be the corresponding eigenvectors of $A$. Then for any vector $C^{T} \in \mathbb{R}^{n}$ which satisfies $C v_{i} \neq 0 . \quad i=1,2, \ldots, p$, the pair $(C, A)$ is observable [13].

Remark 2: The requirement that $A \in \mathbb{R}^{n \times n}$ be cyclic may seem a stringent condition. This condition is satisfied if all eigenvalues of $A$ are distinct, and in the examples given below this condition is satisfied. Moreover, in most chaotic systems, the equations depend on certain parameters, and chaotic behavior is observed when these parameters are in certain ranges. In most cases the eigenvalues depend continuously on these parameters; hence one may choose these parameters accordingly so that the system exhibits chaotic behavior and the matrix $A$ has distinct eigenvalues. Then, by using Lemma 2 , one may find a vector $C^{T} \in \mathbb{R}^{n}$ so that the pair $(C, A)$ is observable.

Remark 3: For a given pair $(C, A)$, whether the observer given by (7) satisfies (10) and Lemma 2 depends on whether the matrix $A_{c}=A-K C$ is stable or not. For observable pairs, by Theorem 1 there always exists a matrix $K$ such that $A_{c}$ is stable. For some pairs $(C, A)$ there may exist a matrix $K$ such that $A_{c}$ is stable, even if the pair is not observable. Such pairs are called "detectable,' and for such pairs the observer given by (7) could still be used [15].

Example 1: (Lorenz system) Consider the Lorenz system given below

$$
\begin{gathered}
\dot{x}_{1}=\sigma\left(x_{2}-x_{1}\right), \\
\dot{x}_{2}=-x_{1} x_{3}+r x_{1}-x_{2}, \\
\dot{x}_{3}=x_{1} x_{2}-b x_{3} .
\end{gathered}
$$

The parameters $\sigma>0, r>0$ and $b>0$ are chosen so that the system exhibits chaotic behavior [2].

We may write (13) in the form given by (4) where $u=\left(\begin{array}{lll}x_{1} & x_{2} & x_{3}\end{array}\right)^{T}$,

$$
A=\left(\begin{array}{ccc}
-\sigma & \sigma & 0 \\
r & -1 & 0 \\
0 & 0 & -b
\end{array}\right), \quad g(u)=\left(\begin{array}{c}
0 \\
-x_{1} x_{3} \\
x_{1} x_{2}
\end{array}\right)
$$

It follows easily that the selection of $y=c_{1} x_{1}+c_{2} x_{3}$ [i.e., $\left.C=\left(\begin{array}{lll}c_{1} & 0 & c_{2}\end{array}\right)\right]$, or $y=c_{1} x_{2}+c_{2} x_{3}$ [i.e., $\left.C=\left(\begin{array}{lll}0 & c_{1} & c_{2}\end{array}\right)\right]$ yields the pair $(C, A)$ observable for almost all values of $c_{1}$ and $c_{2}$, provided that $\left|c_{1}\right| \neq 0,\left|c_{2}\right| \neq 0$. For actual values, (2) should be checked. For $C=\left(\begin{array}{lll}c_{1} & c_{2} & 0\end{array}\right)$ the pair $(C, A)$ is not observable but detectable, i.e., one can easily find matrices of the form $K=\left(\begin{array}{lll}k_{1} & k_{2} & 0\end{array}\right)^{T}$ such that $A-K C$ is stable. In particular, the selection of $y=x_{1}$ [i.e., $C=\left(\begin{array}{lll}1 & 0 & 0\end{array}\right)$, or $y=x_{2}$, [i.e., $C=\left(\begin{array}{lll}0 & 1 & 0\end{array}\right)$ ] makes the pair $(C, A)$ detectable, hence by an appropriate choice of $K$, one may obtain a stable matrix $A-K C$ and use the observer given by (7) for synchronization of chaos.

At this point we compare the observer given by (7) with some synchronization schemes proposed in [2] and [3]. Consider the following system:

$$
\begin{gathered}
\dot{\hat{x}}_{1}=\sigma\left(\hat{x}_{2}-\hat{x}_{1}\right), \\
\dot{\hat{x}}_{2}=-x_{1} \hat{x}_{3}+r x_{1}-\hat{x}_{2}, \\
\dot{\hat{x}}_{3}=x_{1} \hat{x}_{2}-b \hat{x}_{3} .
\end{gathered}
$$

In [2], (16) and (17) are called the response system and in [3], (15)-(17) are called the response system, for the drive system given by (13). Note that here $x_{1}$ is used as the drive signal, hence according to our observer design technique, the output of (13) is $y=x_{1}$. By using the Lyapunov theory, it can be shown that $\lim _{t \rightarrow \infty}\|u(t)-\hat{u}(t)\|=0$, where $u=\left(\begin{array}{lll}x_{1} & x_{2} & x_{3}\end{array}\right)^{T}$ and $\hat{u}=\left(\begin{array}{lll}\hat{x}_{1} & \hat{x}_{2} & \hat{x}_{3}\end{array}\right)^{T}$, see $[2,3]$. Note that (15) -(17) could be written in the form

$$
\dot{\hat{u}}=A \hat{u}+g(\hat{u})+K C(u-\hat{u})+F(\hat{u}) C(u-\hat{u}),
$$

where $A$ and $g$ are given in (14), $K=\left(\begin{array}{lll}0 & r & 0\end{array}\right)^{T}, C=\left(\begin{array}{lll}1 & 0 & 0\end{array}\right)$ and $F(\hat{u})=\left(\begin{array}{ll}0-\hat{x}_{3} & \hat{x}_{2}\end{array}\right)^{T}$. Note that $A-K C$ is a stable matrix with this choice. Hence, the response system given by (15)(17), and hence (18), is similar to the observer given by (7) except for the last term in (18). Without this term Lemma 1 guarantees the local convergence of the error. However, due to the special structure of this term, now we can prove global (exponential) convergence of the error. Due to the special structure of this term, the error equation now becomes

$$
\dot{e}=(A-K C) e+S(t) e,
$$

where

$$
\begin{gathered}
A_{c}=A-K C=\left(\begin{array}{ccc}
-\sigma & \sigma & 0 \\
0 & -1 & 0 \\
0 & 0 & -b
\end{array}\right), \\
S(t)=\left(\begin{array}{ccc}
0 & 0 & 0 \\
0 & 0 & -x_{1}(t) \\
0 & x_{1}(t) & 0
\end{array}\right) .
\end{gathered}
$$

Note that $A_{c}$ is a stable matrix, hence the Lyapunov equation (6) has a symmetric positive definite solution $P$. In particu- 
lar, $P=\operatorname{diag}(\gamma, \beta, \beta)$ is a solution, provided that $\gamma>0, \beta>0$ and $4 \beta>\gamma \sigma$, where diag denotes a diagonal matrix with the specified entries at its diagonal. Note that with this choice, we have $P S(t)=S(t) P$. Hence, by using Lyapunov function $V=e^{T} P e$, differentiating along the error equation (19), we obtain $\dot{V}=-e^{T} Q e$, where $Q$ is given by (6). Therefore (10) is valid for all $e(0) \in \mathbb{R}^{3}$, where

$$
\alpha=\frac{\lambda_{\min }(Q)}{2 \lambda_{\max }(P)} .
$$

For the synchronization of Lorenz system, the following response system has also been proposed by $[2,3,7]$ :

$$
\begin{gathered}
\dot{\hat{x}}_{1}=\sigma\left(x_{2}-\hat{x}_{1}\right), \\
\dot{\hat{x}}_{2}=-\hat{x}_{1} \hat{x}_{3}+r \hat{x}_{1}-\hat{x}_{2}, \\
\dot{\hat{x}}_{3}=\hat{x}_{1} x_{2}-b \hat{x}_{3} .
\end{gathered}
$$

Note that here $x_{2}$ is used as the drive signal, hence according to our observer design technique, the output of (13) is $y=x_{2}$. It could be shown that for this response system, synchronization is achieved. Note that (21) could be written in the form of (18), where $A$ and $g$ are as given by (14), $K=\left(\begin{array}{lll}\sigma & 0 & 0\end{array}\right)^{T}, C=\left(\begin{array}{lll}0 & 1 & 0\end{array}\right)$, and $F(\hat{u})=\left(\begin{array}{lll}0 & 0 & \hat{x}_{1}\end{array}\right)^{T}$. Hence, the response system given by (21) and hence (18), is similar to the observer given by (7) except for the last term in (18). Without this term, Lemma 1 may guarantee the local convergence of the error. With this term, the Lemma 1 is still valid if $\left|\hat{x}_{1}(t)\right| \leqslant M_{1}$ for some $M_{1}>0$, provided that in (9), the left hand side is replaced by $L+M_{1}$. However, due to the form of $F(\cdot)$, we can show that (10) is satisfied for some $M>0$ and $\alpha>0$ provided that the solutions of (13) are bounded. To see that, define $e=\left(\begin{array}{lll}e_{1} & e_{2} & e_{3}\end{array}\right)^{T}$. Then, from (18) it follows that $e_{1}(t)=e^{-\sigma t} e_{1}(0)$, and by using this first in the equation for $e_{3}$, and then in the equation for $e_{2}$, we obtain exponential decay for all error components, provided that $x_{1}(t), x_{2}(t)$, and $x_{3}(t)$ are bounded. Since the Lorenz system exhibits chaotic behavior for the selected set of parameters, its solutions which are of interest to us are bounded; hence this condition is satisfied.

Example 2: (Rössler system) Consider the Rössler system given below:

$$
\begin{gathered}
\dot{x}_{1}=x_{2}+a x_{1}, \\
\dot{x}_{2}=-x_{1}-x_{3}, \\
\dot{x}_{3}=b-c x_{3}+x_{2} x_{3},
\end{gathered}
$$

where the parameters $a>0, b>0$ and $c>0$ are chosen so that the system exhibits chaotic motion, see [2]. This system may be written in the form given by (4) where $u=\left(\begin{array}{lll}x_{1} & x_{2} & x_{3}\end{array}\right)^{T}$,

$$
A=\left(\begin{array}{ccc}
a & 1 & 0 \\
-1 & 0 & -1 \\
0 & 0 & -c
\end{array}\right), \quad g(u)=\left(\begin{array}{c}
0 \\
0 \\
b+x_{2} x_{3}
\end{array}\right) .
$$

It can easily be shown that the selection of $y=c_{1} x_{1}+c_{2} x_{2}+c_{3} x_{3}$ [i.e., $\left.C=\left(\begin{array}{lll}c_{1} & c_{2} & c_{3}\end{array}\right)\right]$ yields the pair
( $C, A)$ observable for almost all $c_{1}, c_{2}$, and $c_{3}$, provided that $\left|c_{1}\right|+\left|c_{2}\right| \neq 0$. For actual values, (2) should be checked. In particular, with the selection of $y=x_{1}$ or $y=x_{2}$, the corresponding pairs $(C, A)$ are observable; hence by choosing the feedback matrix $K$ appropriately, the observer given by (7) may achieve local synchronization. Note that with the selection of $y=x_{3}$, the corresponding pair $(C, A)$ is not even detectable; hence the observer given by (7) could not be used for synchronization for this output.

In the rest of this section we show that the observer given by (7) is robust with respect to measurement noise, i.e., the synchronization error remains bounded for bounded noise. To show this, we assume that the measured output $y$, which is used for synchronization in the observer, is corrupted with noise $n(t)$, hence in (4) we have $y=C u+n$. Then the error equation (8) becomes

$$
\dot{e}=A_{c} e+g(u)-g(\hat{u})-K n(t),
$$

where $A_{c}=A-K C$ is a stable matrix. We assume that the noise $n(t)$ is bounded by some $n_{M}>0$, i.e., $\|n(t)\| \leqslant n_{M}$, $\forall t \geqslant 0$, but arbitrary otherwise. Then the solution of (24) can be written as:

$$
\begin{aligned}
e(t)= & e^{A_{c} t} e(0)+\int_{0}^{t} e^{A_{c}(t-\tau)}\{g[u(\tau)]-g[\hat{u}(\tau)]\} d \tau \\
& -\int_{0}^{t} e^{A_{c}(t-\tau)} K n(\tau) d \tau .
\end{aligned}
$$

Since $A_{c}$ is a stable matrix, it follows that the following is satisfied for some $M>0$ and $\delta>0$ :

$$
\left\|e^{A_{c} t}\right\| \leqslant M e^{-\delta t} .
$$

By using (26) and (5) in (25) and after some simple integration and multiplication by $e^{\delta t}$ we obtain:

$$
\begin{aligned}
\left\|e^{\delta t} e(t)\right\| \leqslant & M\|e(0)\|+\frac{M\|K\| n_{M}}{\delta}\left(e^{\delta t}-1\right) \\
& +\int_{0}^{t} M L\left\|e^{\delta \tau} e(\tau)\right\| d \tau
\end{aligned}
$$

Now by using a generalized form of the Bellman-Gronwall inequality, see e.g., [16, p. 476] and after some simple integration and algebra we obtain:

$$
\|e(t)\| \leqslant A_{1} n_{M}+A_{2} e^{-\delta t}+A_{3} e^{-(\delta-M L) t},
$$

for some constants $A_{1}, A_{2}$, and $A_{3}$, where, in particular, we have

$$
A_{1}=\frac{M\|K\|(\delta-M L+1)}{\delta(\delta-M L)}
$$

Now let us assume that the Lipschitz constant $L$ is sufficiently small so that $\delta-M L>0$, [cf. (9)]. Then it follows from (28) that the synchronization error is also bounded, which implies the stability of the proposed synchronization scheme in the presence of measurement noise. Moreover, asymptotically we have $\|e(t)\| \leqslant A_{1} n_{M}$. Since $A_{1}$ is independent of $n_{M}$, it follows that the smaller the bound $n_{M}$, the 
smaller the synchronization error and in the limit $n_{M} \rightarrow 0$, the synchronization error also asymptotically decays to zero. Hence, we may state that the proposed synchronization scheme is also efficient in this sense in the presence of noise. On the other hand, if the Lipschitz constant $L$ is not sufficiently small but (11) holds, then a similar result holds locally; i.e., if $\|e(0)\|$ and $\|u(t)\|$ are sufficiently small, cf. Lemma 1. The proof of this fact is similar to that of Lemma 1 and is omitted here.

\section{BRUNOWSKY CANONICAL FORM}

In some cases, the local convergence result of the Lemma 1 could be extended to global convergence result, provided that the chaotic system given by (4) has a special form. Assume that the system is in the form (4) with

$$
A=\left(\begin{array}{ccccc}
0 & 1 & 0 & \cdots & 0 \\
0 & 0 & 1 & \cdots & 0 \\
& & \cdot & & \\
& & \cdot & & \\
0 & 0 & 0 & \cdots & 1 \\
0 & 0 & 0 & \cdots & 0
\end{array}\right), \quad g(u)=\left(\begin{array}{c}
0 \\
\cdot \\
\cdot \\
\cdot \\
0 \\
1
\end{array}\right) f(u),
$$

where $f: \mathbb{R}^{n} \rightarrow \mathrm{R}$ is a differentiable function and that $g$ satisfies the Lipschitz property given by (5). The form given by (29) is called the Brunowsky canonical form, and is frequently used in the control of nonlinear systems $[12,14]$. Since the pair $(C, A)$ is observable and $g$ is Lipschitz, the observer given by (7) could be used for local convergence of error, provided that (9) is satisfied. However, it was shown in [12] that for any $L>0$, one can find a feedback matrix $K$, such that (10) is satisfied when the system is in Brunowsky canonical form. Obviously this result still works if the system can be transformed into Brunowsky canonical form by means of a diffeomorphic coordinate transformation. The details can be found in [12]. Here we give a procedure to select the desired $K$, different than the one considered in [12].

For the design of the observer, choose $\lambda_{1}<0$ and $\lambda_{2}=\gamma \lambda_{1}, \lambda_{3}=\gamma^{2} \lambda_{1}, \ldots, \lambda_{n}=\gamma^{n-1} \lambda_{1}$, where $\gamma>1$. Consider the following Vandermonde matrix:

$$
V=\left(\begin{array}{cccc}
\lambda_{1}^{n-1} & \lambda_{1}^{n-2} & \cdots & 1 \\
\lambda_{2}^{n-1} & \lambda_{2}^{n-2} & \cdots & 1 \\
\cdot & & & \\
\cdot & & & \\
\cdot & & & \\
\lambda_{n}^{n-1} & \lambda_{n}^{n-2} & \cdots & 1
\end{array}\right)
$$

It can easily be shown that the feedback matrix $K=\left(\begin{array}{llll}k_{1} & k_{2} & \ldots & k_{n}\end{array}\right)^{T}$ can be appropriately chosen so that

$$
A_{c}=A-K C=V^{-1} \Lambda V,
$$

is satisfied, where $\Lambda=\operatorname{diag}\left(\lambda_{1}, \lambda_{2}, \ldots, \lambda_{n}\right)$. Now consider the error equation given by (8), whose solution can be written as follows:

$$
\begin{aligned}
e(t)= & V^{-1} e^{\Lambda t} V e(0)+V^{-1} \\
& \times \int_{0}^{t} e^{\Lambda(t-\tau)} V B\{f[u(\tau)]-f[\hat{u}(\tau)]\} d \tau,
\end{aligned}
$$

where $B=\left(\begin{array}{llll}0 & 0 \ldots 1\end{array}\right)^{T}$ and

$$
e^{\Lambda t}=\operatorname{diag}\left(e^{\lambda_{1} t}, e^{\lambda_{2} t}, \ldots, e^{\lambda_{n} t}\right)
$$

By taking the max norm $\|\cdot\|_{\infty}$ (see e.g., [14]), we obtain

$$
\begin{aligned}
\|e(t)\|_{\infty} \leqslant & \left\|V^{-1}\right\|_{\infty}\|V\|_{\infty} e^{\lambda_{1} t}\|e(0)\|_{\infty} \\
& +\left\|V^{-1}\right\|_{\infty} \int_{0}^{t} e^{\lambda_{1}(t-\tau)} L\|e(\tau)\|_{\infty} d \tau
\end{aligned}
$$

where we now assumed that (5) is satisfied with the max norm. Note that since in $\mathrm{R}^{n}$ all norms are equivalent, this only affects the Lipschitz constant $L>0$. Also, in (33), we used the matrix norm induced by the max norm. By multiplying both sides of (33) by $e^{-\lambda_{1} t}$, using the BellmanGronwall Lemma, see e.g., [14], we obtain

$$
\|e(t)\|_{\infty} \leqslant\left\|V^{-1}\right\|_{\infty}\|V\|_{\infty} e^{\left(\lambda_{1}+L\left\|V^{-1}\right\|_{\infty}\right) t}\|e(0)\|_{\infty} .
$$

Now simple calculation shows that $\left\|V^{-1}\right\|_{\infty}=G(\gamma)$ for some rational function $G(\cdot)$, provided that $\gamma$ and $\left|\lambda_{1}\right|$ are sufficiently large. Obviously once $\gamma>1$ is chosen sufficiently large, then for any $\alpha>0$ and $L>0$, one can choose $\lambda_{1}$ so that $\lambda_{1}+L\left\|V^{-1}\right\|_{\infty} \leqslant-\alpha$. Hence, (10) is satisfied with $M=\left\|V^{-1}\right\|_{\infty}\|V\|_{\infty}$ and $\alpha$ given by the inequality stated above.

Note that some chaotic systems are already in the form given by (4) and (29) $[17,18]$; hence, the theory presented above can be directly applied for such systems. Some systems may be transformed into this form by a coordinate transformation $z=T(u)$, where $T: \mathbb{R}^{n} \rightarrow \mathbb{R}^{n}$ is a diffeomorphism. The details of finding such a transformation may be found in [12]. Here we emphasize that for some systems this transformation may be linear, i.e., $T(u)=T u$ for some invertible matrix $T \in \mathbb{R}^{n \times n}$, hence the required transformation is quite simple. Now assume that the matrix $A$ given in (4) is in the following form:

$$
A=\left(\begin{array}{cccccc}
* & \alpha_{1} & 0 & 0 & \cdots & 0 \\
* & * & \alpha_{2} & 0 & \cdots & 0 \\
& & & . & & \\
& & & . & & \\
* & * & * & * & \cdots & \\
* & * & * & * & \cdots & \alpha_{n-1}
\end{array}\right),
$$

where the entries given by the asterisk are arbitrary, and $\alpha_{i} \neq 0$ for $i=1,2, \ldots, n-1$. We also assume that $g$ has the form given in (29). Under these conditions there exists a 
linear and invertible transformation $T \in \mathbb{R}^{n \times n}$ such that after the transformation $z=T u$, in the transformed variables the system is given in the form (4) and (29). We note that in this case the required transformation has the form:

$$
T=\left(\begin{array}{cccccc}
1 & 0 & 0 & 0 & \cdots & 0 \\
* & \alpha_{1} & 0 & 0 & \cdots & 0 \\
* & * & \alpha_{1} \alpha_{2} & 0 & \cdots & 0 \\
& & & \vdots & & \\
* & * & * & * & & \alpha_{1} \alpha_{2} \cdots \alpha_{n-1}
\end{array}\right)
$$

hence is always invertible.

Example 2: (revisited) Consider the Rössler system given by (22). Note that $A$ given by (23) is in the form given by (35). By choosing the transformation

$$
\begin{gathered}
z_{1}=x_{1}, \\
z_{2}=a x_{1}+x_{2}, \\
z_{3}=\left(a^{2}-1\right) x_{1}+a x_{2}-x_{3},
\end{gathered}
$$

the Rössler system can be transformed into the form given by (4) and (29), where

$$
\begin{aligned}
f(z)= & -c z_{1}+(c a-1) z_{2}+(a-c) z_{3}-a z_{1}^{2}-a z_{2}^{2} \\
& +\left(a^{2}-1\right) z_{1} z_{2}-a z_{1} z_{3}+z_{2} z_{3}-b .
\end{aligned}
$$

Since the function $f$ given above is differentiable, it follows that the Lipschitz condition (5) is satisfied in any compact region. Since the Rössler system exhibits chaotic behavior for certain values of the parameters $a, b$, and $c$, these chaotic solutions are bounded by a compact region, and in this region (5) is satisfied for some $L>0$. An estimate of $L$ can be found by using $\|D f(z)\|$, see Lemma 1 . Hence by using the technique presented above, an observer for which the synchronization error satisfies (10) can be designed.

Example 3: (Chua's oscillator) We consider the wellknown Chua oscillator which is given in Fig. 1. This circuit is well studied and is known to exhibit many forms of chaotic behavior, see [19,20], and the references therein. The state equations for this circuit are given as

$$
\begin{gathered}
\dot{x}_{1}=-\frac{R_{0}}{L} x_{1}-\frac{1}{L} x_{2}, \\
\dot{x}_{2}=\frac{1}{C_{2}} x_{1}-\frac{G}{C_{2}} x_{2}+\frac{G}{C_{2}} x_{3}, \\
\dot{x}_{3}=\frac{G}{C_{1}} x_{2}-\frac{G}{C_{1}} x_{3}-\frac{1}{C_{1}} f\left(x_{3}\right),
\end{gathered}
$$

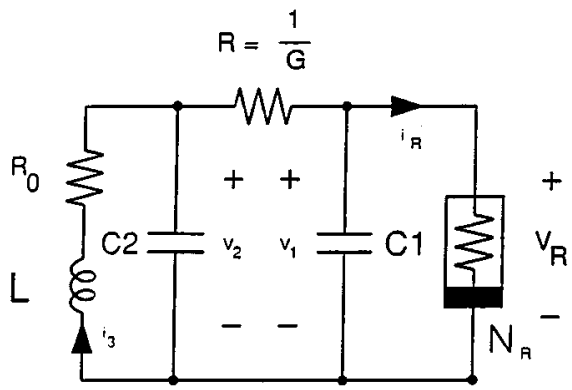

FIG. 1. Chua oscillator.

where $x_{1}=i_{3}, x_{2}=v_{2}, x_{3}=v_{1}, G=1 / R$. The nonlinear resistor $\mathbf{N}_{R}$ is given by the characteristics $i_{R}=f\left(v_{R}\right)$ where the nonlinear function $f: \mathrm{R} \rightarrow \mathrm{R}$ is a three segment piecewise linear function given as, (note that $v_{R}=x_{3}$ ), $f\left(x_{3}\right)=G_{2} x_{3}+0.5\left(G_{1}-G_{2}\right)\left(\left|x_{3}+E\right|-\left|x_{3}-E\right|\right)$ and $G_{1}<0$, $G_{2}<0, E>0$ are some constants, for details see, e.g., [20]. Equations (37) are in the form given by (4), where $g(u)=-\left(\begin{array}{lll}0 & 0 & 1\end{array}\right)^{T}\left(\begin{array}{ll}1 / C_{1} \\ )\end{array}\right)\left(x_{3}\right)$. Note that $g$ is also in the form given by (29) and satisfies (5) globally; in fact $L=\left(1 / C_{1}\right) \max \left\{\left|G_{1}\right|,\left|G_{2}\right|\right\}$. Since matrix $A$ for system (37) has the form given in (35), then by a linear transformation $z=T u$, (37) can be transformed into the form given by (4) and (29). Here $y=x_{1}$ should be chosen as output. Since $g$ satisfies (5) globally, by using the technique given above, the synchronization can be achieved globally, i.e, (10) holds for any initial error $e(0)$.

\section{SIMULATION RESULTS}

In the first simulation example, we considered the Lorenz system given by (13) and the observer given by (7). For Lorenz system, we chose the parameters as $\sigma=10, r=28$ and $b=8 / 3$. For the observer given by (7), we used (14). For the feedback matrix, we chose $K=\left(\begin{array}{lll}-11 / 3 & 253 / 9 & 0\end{array}\right)^{T}$ and the output is chosen as $y=x_{1}$, i.e., $C=\left(\begin{array}{lll}1 & 0 & 0\end{array}\right)$. Note that with this choice, $A-K C$ is a stable matrix. Initial conditions are chosen as $x_{1}(0)=5, \quad x_{2}(0)=-4, \quad x_{3}(0)=5, \quad \hat{x}_{1}(0)=-3$, $\hat{x}_{2}(0)=4, \hat{x}_{3}(0)=-2$, and the resulting simulation results are given in Fig. 2. Note that although this observer may guarantee only local synchronization, in all our simulations we observed convergence. In this particular example we have $\|e(0)\|=13.3$, which is not particularly small.

In the second simulation example, we considered the Rössler system given by (22) and the observer given by (7). For Rössler system, we chose the parameters as $a=0.2$, $b=0.2$, and $c=5$. For the observer given by (7), we used (23). For the feedback matrix, we chose $K=\left(\begin{array}{lll}31 / 5 & 2 & -6\end{array}\right)^{T}$ and the output is chosen as $y=x_{1}$, i.e., $C=\left(\begin{array}{lll}1 & 0 & 0\end{array}\right)$. Note that with this choice, $A-K C$ is a stable matrix. Initial conditions are chosen as $x_{1}(0)=5, x_{2}(0)=-5, x_{3}(0)=-4, \hat{x}_{1}(0)=-5$, $\hat{x}_{2}(0)=5, \hat{x}_{3}(0)=4$, and the resulting simulation results are given in Fig. 3. Note that although this observer also guarantees only local synchronization, as in the Lorenz system, in all our simulations we observed convergence. In this particular example we have $\|e(0)\|=16.2$, which is not particularly small.

In the third simulation, we considered Chua's oscillator given in Fig. 1. In the simulations we chose $R_{0}=0$, which 

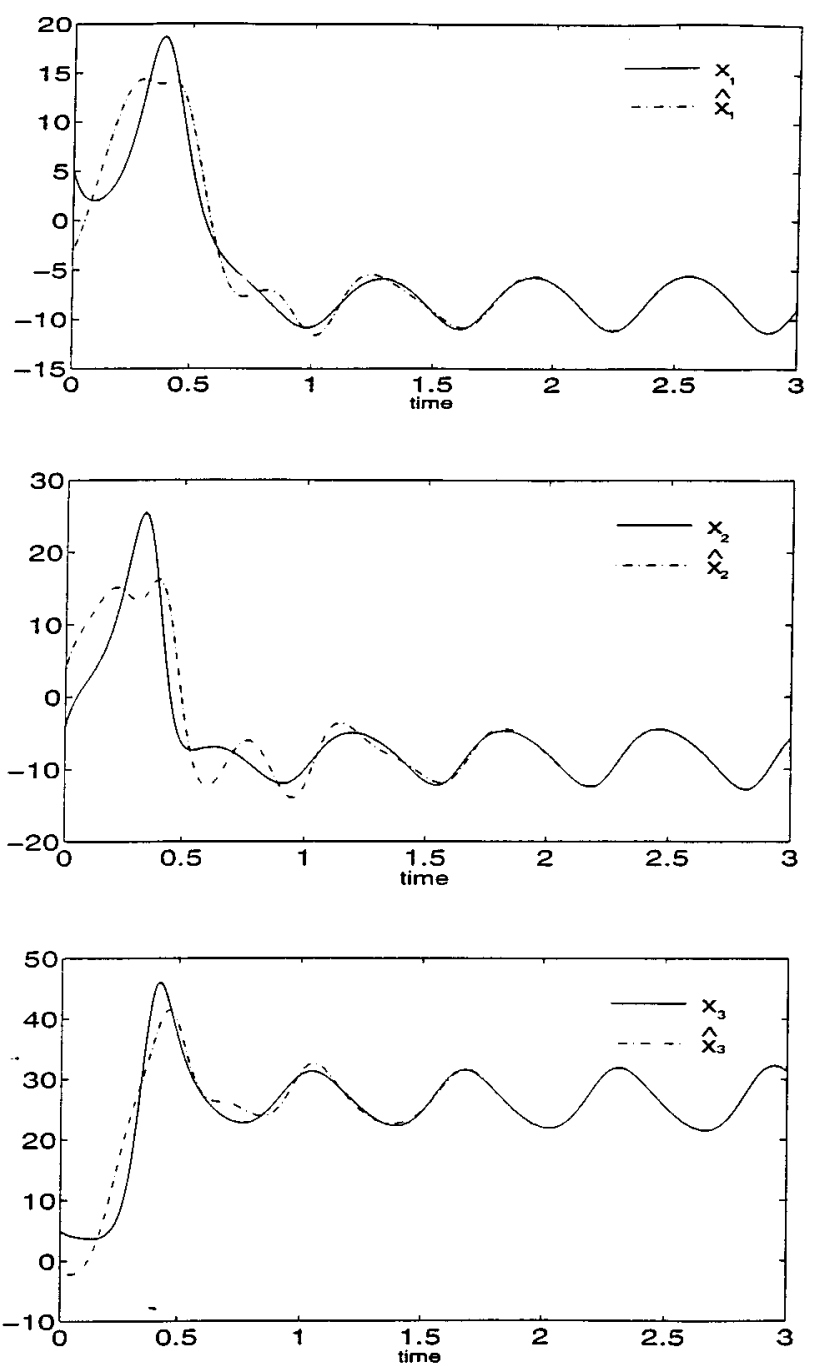

FIG. 2. Drive and observer states for the Lorenz system. For $i=1,2,3, x_{i}$, and $\hat{x}_{i}$ denote the drive and the observer states, respectively.

does not affect the chaotic behavior, but simplifies Eqs. (34), [20]. For actual values of the parameters to observe chaotic behavior, see $[19,20]$. For these actual values, the parameters in (34) may be too large, especially the Lipschitz constant in (5) may be in the range of $10^{6}$, which causes problems in determining the observer. To overcome this difficulty, we first scaled the time and used $\tau=\left(G / C_{2}\right) t$ as the new independent variable and also scaled the variable $x_{1}$ by $1 / G$. After these changes, (34) now becomes

$$
\begin{gathered}
\dot{x}_{1}=-\beta x_{2}, \\
\dot{x}_{2}=x_{1}-x_{2}+x_{3}, \\
\dot{x}_{3}=\alpha x_{2}-\alpha x_{3}-\frac{\alpha}{G} f\left(x_{3}\right),
\end{gathered}
$$

where $\alpha=\left(C_{2} / C_{1}\right)$ and $\beta=\left(C_{2} / L G^{2}\right)$. Following [21], we choose the parameters as $G_{1}=-0.8, G_{2}=-0.5, \alpha=8, \beta=11$, $E=1$, and $G=0.7$. As is shown in [21], with these parameters, the equations given above exhibit a double scroll type chaotic behavior, see [20]. For the feedback matrix, we chose
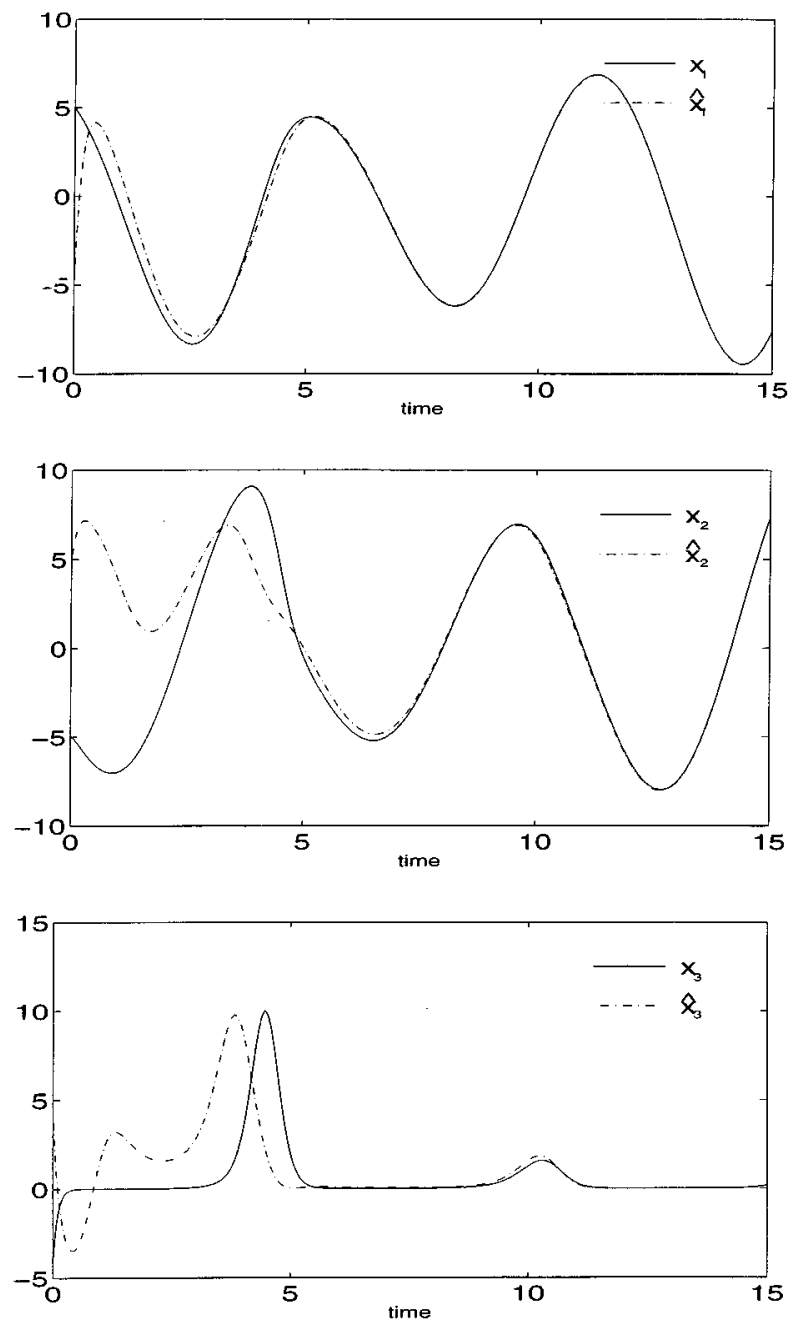

FIG. 3. Drive and observer states for the Rössler system. For $i=1,2,3, x_{i}$, and $\hat{x}_{i}$ denote the drive and the observer states, respectively.

$K=(121-2800 / 11-4512 / 11)^{T}$ and the output is chosen as $y=x_{1}$, i.e., $C=\left(\begin{array}{lll}1 & 0 & 0\end{array}\right)$. Note that with this choice, $A-K C$ is a stable matrix. Note that here we used the procedure given in Sec. III, in particular, we chose $\lambda_{1}=-10, \lambda_{2}=-30$, $\lambda_{3}=-90$ in the Vandermonde matrix given by (30) and $K$ is determined by (31). Initial conditions are chosen as $x_{1}(0)=0.1, \quad x_{2}(0)=0.1, \quad x_{3}(0)=0.1, \quad \hat{x}_{1}(0)=2, \quad \hat{x}_{2}(0)=2$, $\hat{x}_{3}(0)=2$, and the resulting simulation results are given in Fig. 4. Note that in this case, according to the theory presented in Sec. III, global convergence is guaranteed. We also note that in Fig. 4 the horizontal axis denote the scaled time $\tau$. Since $\tau=\left(G / C_{2}\right) t$, and in the Chua's oscillator the capacitors are normally chosen in nanofarad range, see $[19,20]$; Fig. 4 shows that convergence is achieved in nanoseconds range.

In the last simulation we again considered the Rössler system given by (22) and the observer given by (7). This time we assumed that the measurement is corrupted by a noise, i.e., in (7) we assumed that $y=x_{1}(t)+n(t)$. The noise $n(t)$, which is generated by computer, is uniformly distributed and zero mean noise and its magnitude is bounded by $10^{-1}$, i.e., $n_{M}=10^{-1}$ in (28). The system parameters, initial conditions, and the feedback gain $K$ are selected as in the 

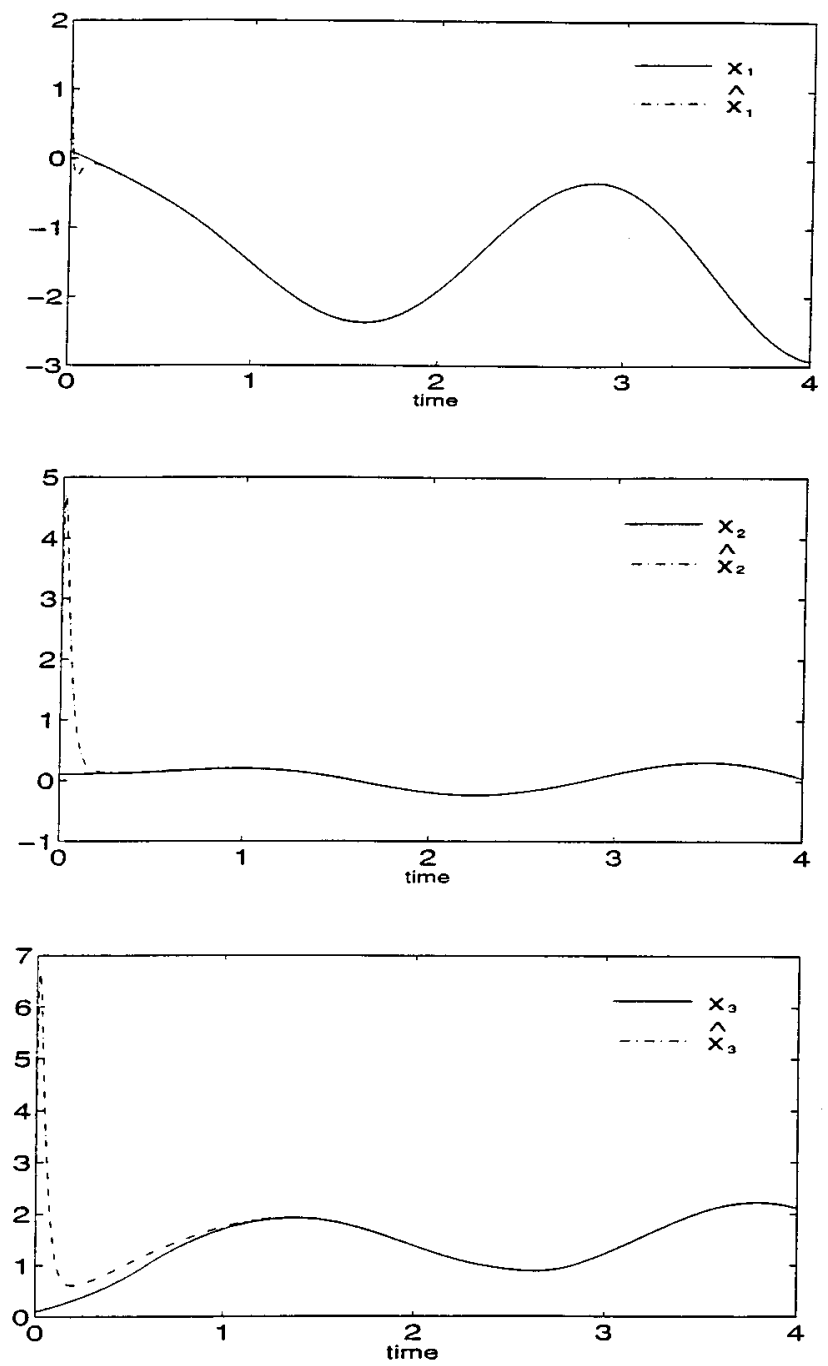

FIG. 4. Drive and observer states for the Chua oscillator. For $i=1,2,3, x_{i}$, and $\hat{x}_{i}$ denote the drive and the observer states, respectively.

second simulation given above. The simulation results are given in Fig. 5. As can be seen from Fig. 5, the effect of the noise in the synchronization is considerably small.

\section{CONCLUSIONS}

Most of the synchronized chaotic systems proposed in the literature consist of two parts: a drive system which generates the chaotic signals, and a response system. Some signals called drive signals are generated by the drive system and are used in the response system to synchronize the common signals of both systems. In most of the cases, once the drive system is given, the determination of the response system and the drive signals are not systematic and one scheme proposed for a particular drive system could not be easily generalized to an arbitrary chaotic drive system.

In this paper we considered the observer based synchronization of chaotic systems. Observers are widely used in systems and control theory to estimate the states of a given system; hence they may naturally be used in the synchronization of chaotic systems. In this approach, once the chaotic drive system is given in a form [see (4)], then the response
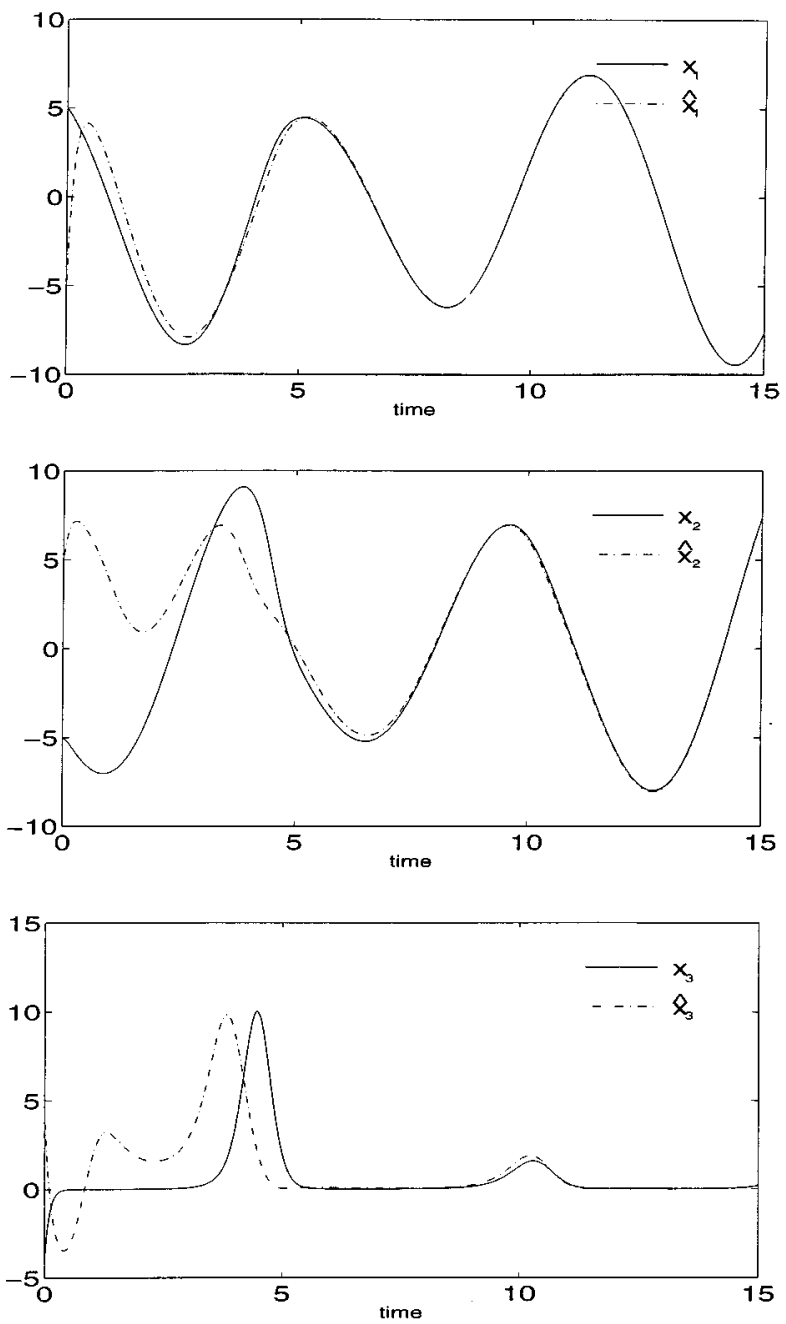

FIG. 5. Drive and observer states for the Rössler system in the presence of noise. For $i=1,2,3, x_{i}$, and $\hat{x}_{i}$ denote the drive and the observer states, respectively.

system could be chosen as an observer, [see (7)], provided that the output corresponding to the selected drive signal satisfies some conditions (i.e., observability or detectability, see Theorem 1, Remark 3). These conditions are not very restrictive and are satisfied by most of the chaotic systems, (see Lemma 2, Remark 2). Then we stated a general result on the local synchronization of the drive system and the observer (see Lemma 1). We showed that the proposed scheme is robust with respect to measurement noise under certain conditions. We also stated a global convergence result, provided that the system could be transformed into a special form. We also showed that some of the existing schemes for the synchronization of chaos are related to the observer based synchronization proposed in this paper. We also presented some numerical simulation results for the Lorenz, Rössler systems, and Chua's oscillator, which are known to exhibit many forms of chaotic behavior.

We note that the form of the observer given in this paper is not the only possible form. There are many observer design techniques and some of them may give better results in the synchronization of chaotic systems. This point requires further research and the results will be presented elsewhere. 
[1] L. M. Pecora and T. L. Carroll, Phys. Rev. Lett. 64, 821 (1990).

[2] L. M. Pecora and T. L. Carroll, Phys. Rev. A 44, 2374 (1991).

[3] K. M. Cuomo and A. V. Oppenheim, Phys. Rev. Lett. 71, 65, (1993).

[4] K. M. Cuomo, A. V. Oppenheim, and S. H. Strogatz, IEEE Trans. Circuits Syst. 40, 626, (1993).

[5] K. S. Halle, C. W. Wu, M. Itoh, and L. O. Chua, Int. J. Bifurcation Chaos 3, 469, (1993).

[6] L. Kocarev, K. S. Halle, K. Eckert, and L. O. Chua, Int. J. Bifurcation Chaos 2, 709, (1992).

[7] R. He and P. G. Vaidya, Phys. Rev. A 46, 7387, (1992).

[8] M. J. Ogorzalek, IEEE Trans. Circuits Syst. 40, 693, (1993).

[9] L. O. Chua, L. Kocarev, and K. Eckert, Int. J. Bifurcation Chaos 2, 705, (1992).

[10] F. E. Thau, Int. J. Control 17, 3, (1973).

[11] E. A. Misawa and J. K. Hedrick, Trans. ASME J. Dynamic Syst. Measur. Control 111, 344, (1989).
[12] G. Ciccarella, M. Dalla Mora, and A. Germani, Int. J. Control 57, 537, (1993).

[13] T. Kailath, Linear Systems (Prentice-Hall, Englewood Cliffs, 1980).

[14] M. Vidyasagar, Nonlinear Systems Analysis, 2nd ed. (PrenticeHall, Englewood Cliffs, 1993).

[15] W. M. Wonham, Linear Multivariable Control, a Geometric Approach, 3rd ed. (Springer-Verlag, New York, 1985).

[16] F. M. Callier and C. A. Desoer, Linear System Theory (Springer-Verlag, New York, 1991).

[17] A. Tesi, A. De Angeli, and R. Genesio, Int. J. Bifurcation Chaos 4, 1675, (1994).

[18] A. A. Alexeyev and V. D. Shalfeev, Int. J. Bifurcation Chaos 5, 551, (1995).

[19] M. P. Kennedy, IEEE Trans. Circuits Syst. part 1, 40, 657 (1993).

[20] L. O. Chua, C. W. Wu, A. Huang, and G. Q. Zhong, IEEE Trans. Circuits Syst. part 1, 40, 732 (1993).

[21] R. Genesio and A. Tesi, Automatica 28, 531 (1992). 